\title{
Propuesta de diseño de un sistema continuo para un fotobiorreactor airlift a escala laboratorio
}

\section{Proposal of design of a continuous system for an airlift photobiorreactor in laboratory scale}

\author{
págs. 94-105 \\ Grupo de investigación: Biotecfua \\ Línea de investigación: Biotecfua \\ Diego Rubio Fernández• Diana K. Guzmán•• Cristian M. Gutiérrez••• Juan A. Sandoval•..•
}

\section{RESUMEN}

Las microalgas han sido usadas como fuente de productos bioquímicos con un alto valor agregado. Esto plantea la necesidad de realizar procesos que sean rentables y con una alta calidad. El uso de sistemas continuos para estos procesos es una buena opción. El objetivo de esta investigación es realizar una propuesta de diseño de un sistema continuo para un fotobiorreactor Airlift a escala laboratorio, manejando variables de diseño como los flujos de entrada y salida, el diámetro de la manguera, el tiempo de retención de los tanques de almacenamiento y la potencia de las bombas. El método matemático parte de los balances de masa y energía, que permiten relacionar estas variables con valores ya definidos para este sistema; como resultado, además de solucionar estas variables de diseño, permitió definir y delimitar las diferentes partes y componentes del sistema continuo.

Palabras clave: microalgas, sistema continuo, variables de diseño, balances de masa, balances de energía.

\begin{abstract}
Microalgae has been used as a source for biochemical compounds with a high added value. It implies the need for developing profitable processes with high quality. A continuous system is a good option to be used to improve that kind of process. The objective of this research was to propose a design of a continuous system for an Airlift photobioreactor in a laboratory scale, by means of design variables such as inlet and outlet flows, diameter of the hosepipe, retention time in the storage tanks and power of the pump. The mathematical method begins with mass and energy balances that allowed relating these variables with values already defined for this system. As a result, with these balances not only was it possible to solve these design variables, but it also allowed defining and delimiting the different parts and components of the continuous system.
\end{abstract}

Key words: microalgae, continuous system, design, design variables, mass balances, energy balances.

-Biólogo. Docente-Investigador. Universidad de América. Correo: diego.rubio@profesores.uamerica.edu.co

-•Ingeniera Química. Universidad de América. Correo: diana.guzman2@estudiantes.uamerica.edu.co

..• Ingeniero Químico. Universidad de América. Correo: cristian.gutierrez2@estudiantes.uamerica.edu.co

...• Ingeniero Químico. Docente Universidad de América. Correo: juan. sandoval@profesores.uamerica.edu.co 
LÍNEA DE INVESTIGACIÓN: BIOTECFUA

\section{INTRODUCCIÓN}

Las microalgas son organismos unicelulares microscópicos empleados como fuente de una gran variedad de aplicaciones que van desde el uso de la biomasa hasta la producción de compuestos con un alto valor agregado (Bruno et al., 2015). Entre las estrategias de operación para mejorar la producción y el rendimiento de las microalgas se encuentra el modo continuo (Shih-Hsin et al., 2014), el cual permite un mayor rendimiento en comparación con un sistema por lotes. Factores como el alto costo de mantenimiento del cultivo debido a la baja concentración celular, bajas productividades y una calidad variable de los productos hacen que el sistema por lotes no sea viable (Ganuza \& Izquierdo, 2007; Bruno et al., 2015). Además, el sistema continuo permite manejar el cultivo de forma exponencial mientras que en el sistema por lotes la microalga debe pasar por todas las etapas de crecimiento (Fig. 1).

A nivel de investigación, se ha estudiado la producción de microalgas mediante sistema por lotes, principalmente en la obtención de biocombustibles, tales como bioetanol o biodiesel (Caicedo \& Rodríguez, 2016; Ángel \& Pimienta, 2013).

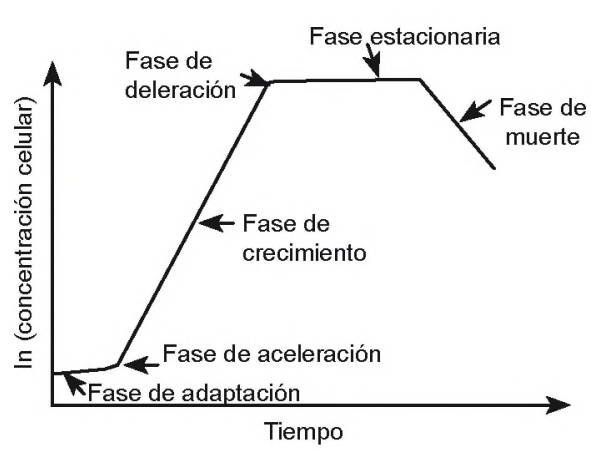

a)

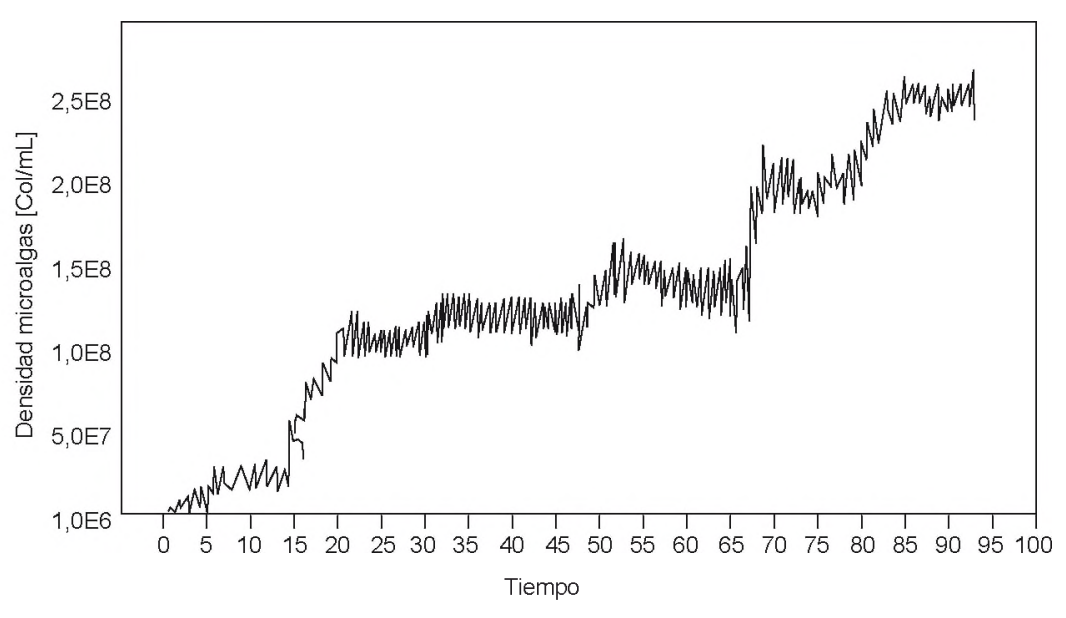

b)

Figura 1. Curva de crecimiento de un microrganismo para un sistema por lotes (a) y para un sistema continuo (b) ${ }^{1}$

Sin embargo, las investigaciones sobre la producción de microalgas mediante un sistema continuo son muy pocas, por lo cual, no hay suficiente información acerca de este tipo de operación.

Esta investigación tiene como objetivo proponer un diseño de un sistema continuo para un fotobiorreactor Airlift a escala laboratorio, con el fin de encontrar aquellos parámetros que permitan una operación viable y garantice un producto de calidad.

1 Modificado de: (a). DORAN, Pauline M. y GARCÍA LABIANO, Francisco J. Principios de Ingeniería de los Bioprocesos. España: Acribia, 1995. (b). CHO, Dae-Hyun, et al. Influence of limiting factors on biomass and lipid productivities of axenic Chlorella vulgaris in photobioreactor under chemostat cultivation. En: BIORESOURCE TECHNOLOGY. 22 marzo del 2016 vol. 211, p. 367 


\section{MATERIALES Y MÉTODOS}

La línea de investigación Biotecfua ya cuenta con un fotobiorreactor Airlift a escala laboratorio de cilindros concéntricos. Este fotobiorreactor funciona por lotes, lo cual este es el punto de partida de la propuesta del sistema continuo.

\section{Variables de diseño}

Las variables de diseño que se tuvieron en cuenta se dividieron en dos grupos: las variables geométricas y las variables de proceso que son específicas del funcionamiento de un sistema continuo.

\section{Propuestas de diseño}

Se plantearon dos propuestas de diseño para el fotobiorreactor Airlift. Con el fin de tener una propuesta definitiva de diseño, la propuesta final se seleccionó por medio de un análisis cualitativo y cuantitativo.

- Cálculos. Los cálculos se realizaron respecto de cada una de las variables seleccionadas para el diseño.

Flujos de entrada y salida del fotobiorreactor. Para el cálculo de los flujos se relacionaron estas variables con datos obtenidos en otros trabajos, por medio de balances de biomasa y global del fotobiorreactor, las cuales se simplificaron en las ecuaciones 1 (Abalde, 1995), 2 y 3 (Doran, 1995)

$$
\begin{gathered}
\mathrm{F}_{\text {out }}=\mu \times \mathrm{V}_{\mathrm{L}} \\
d=\frac{1}{\tau}=\mu \\
F_{\text {in }}=\frac{\rho \text { out } \times F_{\text {out }}}{\rho \text { in }}
\end{gathered}
$$

Aquí, $F_{\text {out }}$ es el flujo de salida del fotobiorreactor; $\mu$ velocidad de crecimiento del microorganismo; $V_{L}$ volumen de operación del fotobiorreactor; $d$ tasa de dilución; $\tau$ tiempo de retención en el fotobiorreactor; $F_{i}$ Flujo de entrada del fotobiorreactor; $\rho_{\text {out }}$ densidad del flujo de salida y $\rho_{\text {in }}$ densidad del flujo de entrada. Los valores de densidad se obtuvieron por medio de una prueba con picnómetro con $50 \mathrm{~mL}$ de medio de cultivo y $50 \mathrm{~mL}$ de biomasa y utilizando la ecuación 4 (Osorio \& Zapata, 2004).

$$
\rho=\frac{P_{\text {lleno }}-P_{\text {vacio }}}{V_{\text {picnómetro }}}
$$

Diámetro de manguera. El diámetro de manguera fue definido por dos criterios: el primero, la oferta en el mercado nacional y disponibilidad de accesorios. El segundo criterio, su relación con el estrés celular por medio del análisis del número de Reynolds Ecuación 5, trabajo de cizalla Ecuación 6 y velocidad de deformación de cizalla Ecuación 8 (Steffe \& Daubert, 2006). 
LÍNEA DE INVESTIGACIÓN: BIOTECFUA

$$
\begin{gathered}
\operatorname{Re}=\frac{\rho_{x} v D}{\mu} \\
W S=\frac{k f v^{2}}{2} \\
W=\Sigma W s \\
\gamma_{\text {avg }}=\frac{2 v}{D_{x}}
\end{gathered}
$$

Donde $R e$, es número de Reynolds; $v$, es velocidad; $\rho_{x}$, densidad del fluido en la tubería; $k_{f}$ coeficiente de resistencia con fluidos biológicos; $D_{x}$ diámetro de la tubería; $\eta$, viscosidad absoluta; $W_{s}$, trabajo de cizalla en accesorios y tubería; $W$, trabajo de cizalla en el sistema y $\gamma_{\text {avg, }}$ velocidad de deformación de cizalla.

Tiempo de retención en los tanques. Se fijó un tiempo de funcionamiento de 15 días, debido a que en ese tiempo la microalga Chlorella vulgaris crece en el fotobiorreactor (Caicedo \& Rodríguez, 2016). Este tiempo define el tamaño y las dimensiones de los dos tanques, que se calcularon por medio de las ecuaciones 9 a 13 (Montouto, 2010). Se seleccionaron dos tanques, porque en uno se realiza el cultivo (fotobiorreactor) y en el otro la separación del medio de cultivo de la microalga, cuando ya ha alcanzado la fase estacionaria (tanque de almacenamiento de biomasa). Cabe mencionar que se sobredimensionaron los tanques con un factor de seguridad del $20 \%$.

$$
\begin{gathered}
\mathrm{V}_{\mathrm{L}}=\mathrm{t}_{\mathrm{fu}} \mathrm{F}_{\text {in }} \\
V_{\text {Total }}=\frac{V_{L}}{0,8} \\
\sqrt[3]{\frac{V_{\text {Total }}}{2 k \pi}}=R \\
\mathrm{H}=2 \mathrm{kR} \\
H_{\text {operación }}=\frac{V_{\text {operación }}}{R^{2} \pi}
\end{gathered}
$$

Donde $V_{L}$ es el volumen total necesario para cumplir con el tiempo de operación; $t_{f u}$ tiempo de retención en los tanques; $V_{\text {Total, }}$ volumen del tanque; $k$, constante de relación altura/diámetro; $R$, radio y $H$, altura. Si se desea calcular el volumen máximo del sistema, se utiliza la Ecuación 14.

$$
V_{\text {máx }}=V_{o p . f b}+V_{o p . b i o}
$$


En la Ecuación 14, $V_{\text {op.tfb }}$ es volumen de operación del fotobiorreactor y $V_{\text {op.bio, }}$ es volumen de operación de la biomasa en el tanque.

Potencia de las bombas. Se parte de un balance de energía por medio de la Ecuación 15. Se divide el sistema en dos zonas: carga y descarga, para definir los puntos de referencias necesarios en el balance de energía. Estas zonas de observan en la figura 2. Posteriormente, se calcula el número de Reynolds, las pérdidas menores, la carga total sobre la bomba y finalmente la potencia que debe transmitir la bomba al fluido. Esto se realizó por medio de una hoja de cálculo de Excel que resuelve las ecuaciones 15 a 27 (Mott; Enriquez, \& Leon, 2006).

$$
\begin{gathered}
h_{a}=\frac{P_{2}-P_{1}}{\gamma}+\frac{v_{2}^{2} v_{1}^{2}}{2 g}+Z_{2}-Z_{1}+h_{L} \\
V_{2}=\frac{F_{\text {in }}}{A_{t}} \\
A_{t}=\frac{\pi D^{2}}{4} \\
h_{i}=\Sigma h_{n} \\
h_{n}=K\left(\frac{v^{2}}{2 g}\right) \\
P_{o}=F_{x} \gamma h_{a}
\end{gathered}
$$

Donde $\gamma$ es peso específico del fluido; $g$ gravedad de la Tierra; $h_{a}$ carga total sobre la bomba; $F_{1}, P_{2}$ presiones manométricas en los puntos 1 y $2 ; v_{1}, v_{2}$ velocidades del fluido en los puntos 1 y 2 ; $z_{1}, z_{2}$ elevaciones de los puntos 1 y $2 ; h$, pérdida total de energía por unidad de peso del fluido en movimiento; $h_{n}$ pérdidas de energía por ficción; $A_{t}$ área transversal de la manguera; $P_{o}$ potencia de la bomba; $F_{x}$ Flujo volumétrico; y $h_{a}$ pérdida de energía por accesorios.

Para el cálculo de los coeficientes de rozamiento, involucrados en el cálculo de pérdida de energía por accesorios, es necesario emplear dos tipos de ecuaciones: unas para fluidos no biológicos, como las Ecuaciones 21, 25 y 27 (Mott; Enríquez, \& León, 2006), mientras que otras para los fluidos biológicos: 22 a 26 (Steffe \& Daubert, 2006).

$$
\begin{gathered}
K=\frac{L_{e}}{D} \oslash f_{t} \\
k_{f}=\frac{K_{1}}{R_{e}}+K_{\propto}\left(1+\frac{1}{D}\right)
\end{gathered}
$$


LÍNEA DE INVESTIGACIÓN: BIOTECFUA

$$
\begin{gathered}
k_{f}=\frac{K_{1}}{R_{e}}+K_{\propto} \\
k_{f}=\left(1,2+\frac{160}{R_{e}}\right)\left(\left(\frac{D_{1}}{D_{2}}\right)^{4}-1\right) \\
K=f_{t}\left(\frac{L}{D}\right) \\
f_{t}=\frac{16}{R e} \\
f_{t}=\frac{64}{R e}
\end{gathered}
$$

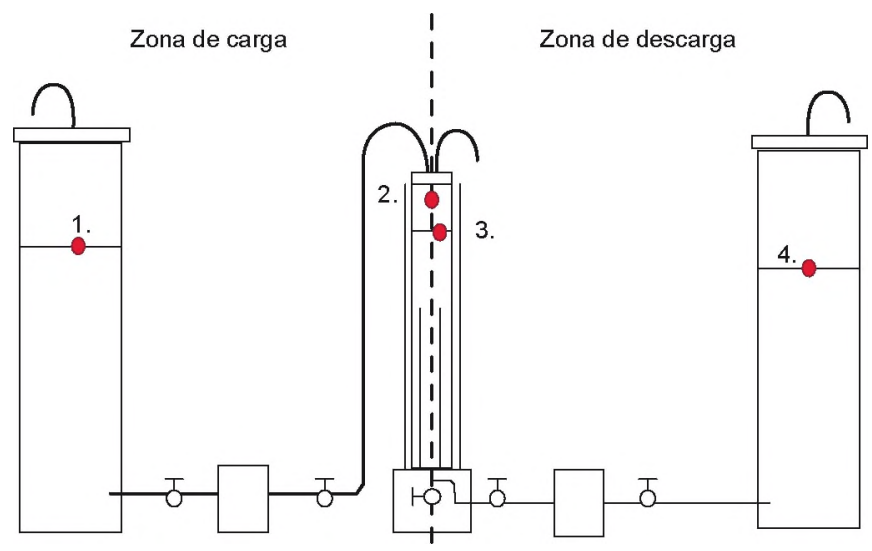

Figura 2. Puntos de cálculo y zonas del sistema continuo.

En donde $K$ es coeficiente de resistencia; $\frac{L_{a}}{2}$ es la relación de longitud equivalente a diámetro, propia del accesorio; $f_{t}$ factor de fricción; $k_{f}$ coeficiente de resistencia con fluidos biológicos; los valores de $k_{1}$ y $k_{\infty}$ se encuentran en el libro de Steffe (2006).

Como se observa, en las ecuaciones 15 al 27 se deben conocer las viscosidades dinámicas y cinemáticas del medio de cultivo y la biomasa. Debido a esto fue necesario hacer una prueba de laboratorio empleando un viscosímetro Cannon-Fenske 100. Luego se calculó la viscosidad con las Ecuaciones 28 y 29; para el peso específico se usó la Ecuación 30.

$$
\begin{gathered}
V_{x}=0,01446 \cdot\left(t-t_{H C}\right) \\
\eta_{\mathrm{x}}=v_{x} \rho_{x}
\end{gathered}
$$




$$
\gamma=\rho_{x} g
$$

Donde: $v_{x}$ es Viscosidad cinemática; $t$ tiempo que tarda el líquido para fluir entre las marcas de referencia; $t_{H C}$ tiempo de corrección de energía cinemática y $\eta_{x}$ viscosidad dinámica.

\section{RESULTADOS Y DISCUSIÓN}

\section{Variables de diseño}

Las variables seleccionadas fueron: flujo de entrada y salida del fotobiorreactor; diámetro de la manguera; tiempo de retención en los tanques de almacenamiento y potencia de las bombas. Esta selección se hizo debido a que son variables independientes y se pueden relacionar con otros parámetros y condiciones por medio de balances de materia y energía.

\section{Propuestas de diseño}

De acuerdo con la metodología se plantearon dos propuestas de diseño.

- Primera Propuesta. Se define un tiempo de funcionamiento del sistema continuo de 15 días, con dos bombas: la primera bomba dirige el medio de cultivo hacia el fotobiorreactor (tanque 1), mientras que la segunda lleva la biomasa del fotobiorreactor hacia el tanque de almacenamiento de biomasa (tanque 2).

El sistema cuenta con una válvula de bola, una llave de doble tipo con cierre bilateral, un adaptador de manguera; la salida del tanque de alimentación al fotobiorreactor es lateral, sin embargo, la entrada al tanque de almacenamiento de biomasa está en la parte superior de este.

- Segunda propuesta. Se establece un tiempo de funcionamiento de 15 días, basado en la curva de crecimiento de la microalga (Ver figura 1). El sistema cuenta con una válvula de bola, una llave de doble tipo con cierre bilateral, una llave de cierre en el tanque de biomasa, dos bombas; las mangueras van conectadas a la salida posterior del tanque de almacenamiento y a la parte superior del tanque de recolección de biomasa. Además se plantea el uso de soportes y microfiltros de aire en los tanques de almacenamiento.

\section{Cálculos}

- Flujos de entrada y salida del fotobiorreactor. Por medio de la velocidad de crecimiento de la microalga Chlorela vulgaris en fase exponencial (tabla 1) y el volumen total de operación del fotobiorreactor, se calcularon los flujos de salida que se encuentran en la tabla 2 , empleando la Ecuación 3. 
LÍNEA DE INVESTIGACIÓN: BIOTECFUA

Tabla 1.

Velocidades de crecimiento específico para microalga Chlorella vulgaris.

\begin{tabular}{lc}
\hline \multicolumn{1}{c}{ Referencia } & Velocidad de crecimiento (s-1) \\
\hline (Romdhane, 2012) & $5,0 \mathrm{E}-06$ \\
& $4,3 \mathrm{E}-07$ \\
& $1,1 \mathrm{E}-06$ \\
(López, Malagón, \& Torres, 2013) & $6,1 \mathrm{E}-07$ \\
& $6,5 \mathrm{E}-07$ \\
\hline
\end{tabular}

Como se observa en la tabla 1, las velocidades de crecimiento de la microalga son variables. Esto se debe a las diversas condiciones de cultivo; la cantidad y el tiempo del inóculo que se agregó en cada una de las investigaciones.

Tabla 2.

Flujos de salida del fotobiorreactor

\begin{tabular}{cc}
\hline Velocidad de crecimiento $(\mathbf{s}-\mathbf{1})$ & Flujo de salida $\left(\mathbf{m}^{3} / \mathbf{s}\right)$ \\
\hline $5,0 \mathrm{E}-06$ & $8,0 \mathrm{E}-09$ \\
$4,3 \mathrm{E}-07$ & $1,8 \mathrm{E}-09$ \\
$1,1 \mathrm{E}-06$ & $1,0 \mathrm{E}-09$ \\
$6,1 \mathrm{E}-07$ & $9,7 \mathrm{E}-10$ \\
$6,5 \mathrm{E}-07$ & $6,8 \mathrm{E}-10$ \\
\hline
\end{tabular}

De acuerdo con los valores de la tabla 2 el flujo de salida está entre $6,8 \mathrm{E}-10 \mathrm{~m}^{3} / \mathrm{s} \mathrm{a} 8,0 \mathrm{E}-9 \mathrm{~m} / \mathrm{s}$. Al comparar estos valores con los del trabajo realizado por Romdhane (2012), se observó que los flujos para estos tipos de sistemas continuos son bajos, esto se debe a la baja velocidad de crecimiento de la microalga y a la escala del fotobiorreactor. Para el flujo de entrada se necesitaron las densidades del medio de cultivo y la biomasa que se encuentran en la tabla 3. Ecuación 1.

Tabla 3.

Densidades y flujo de entrada al fotobiorreactor

\begin{tabular}{llc}
\hline \multicolumn{1}{c}{ Variable } & \multicolumn{1}{c}{ Valor } & Unidades \\
\hline Densidad del medio de cultivo & 933,721 & $\mathrm{~kg} / \mathrm{m}^{3}$ \\
Densidad de la biomasa & 1000,176 & $\mathrm{~kg} / \mathrm{m}^{3}$ \\
Flujo de entrada & $7,3 \mathrm{E}-10-8,6 \mathrm{E}-09$ & $\mathrm{~m} / \mathrm{s}$ \\
\hline
\end{tabular}

Como se evidencia en la tabla 3, la densidad a la salida será mayor que a la de entrada; esto se debe principalmente a que la microalga al consumir los nutrientes genera una mayor cantidad 
de masa a la salida del fotobiorreactor, razón por la cual para mantener el fotobiorreactor en condiciones estables el flujo de entrada tiene que ser mayor al de salida.

- Diámetro. Los diámetros seleccionados de las mangueras son $1 / 4$ in $(6,35 \mathrm{~mm})$ interno y $3 / 8$ in $(9,53 \mathrm{~mm})$ externo. Esto se debe a dos razones: La primera es la disponibilidad de estas medidas en las mangueras siliconadas utilizadas en este tipo de procesos, además que la mayoría de conexiones de estas mangueras a las bombas, válvulas y accesorios están para 1/4 in y $3 / 8$ in de diámetro. La segunda está relacionada con los cálculos del número de Reynolds, trabajo de cizalla y velocidad de deformación de cizalla, cuyos valores se encuentran en la tabla 4.

Tabla 4.

Diámetro y estrés celular

\begin{tabular}{|c|c|c|c|}
\hline \multirow{2}{*}{ Variable } & \multicolumn{2}{|c|}{ Valor } & \multirow{2}{*}{ Unidades } \\
\hline & Tubería 1 & Tubería 2-3 & \\
\hline Numero de Reynolds & 0,72 & 1,45 & \\
\hline Velocidad de deformación de cizalla & $9,97 \mathrm{E}-3$ & 7,97E-2 & s-1 \\
\hline Fuerza de cizalla del sistema & $1,95 \mathrm{E}-4$ & & $\mathrm{~J} / \mathrm{kg}$ \\
\hline
\end{tabular}

El número de Reynolds y velocidad de deformación de cizalla para un diámetro de 1/4 in son bajos en comparación con la investigación realizada por Michels (2010) en la que se presentó muerte celular por fuerza de cizalla a números de Reynolds entre 9,3E3 y 2,3E4. Por otro lado, la velocidad de deformación dependerá de las condiciones en las cuales se trabaje la microalga; de acuerdo con esto se encontraron valores de 2,2 s $\mathrm{s}^{-1}$ a $22 \mathrm{~s}^{-1}$. Por lo tanto, se puede afirmar que para el sistema propuesto en este trabajo de investigación la microalga no sufrirá ningún daño durante el transporte del fotobiorreactor al tanque de almacenamiento de biomasa.

- Tiempo de retención en los tanques. Para el cálculo de este parámetro, se empleó el flujo de entrada al tanque 1. No se observaron diferencias apreciables en las dimensiones de cada uno de los tanques, por lo cual se asumieron los mismos valores. En la Tabla 5 se encuentran los resultados.

Tabla 5.

Dimensiones de los tanques

\begin{tabular}{lcc}
\hline \multicolumn{1}{c}{ Parámetros } & T = 15 dias & Unidades \\
\hline Volumen total & 0,014 & $\mathrm{~m}^{3}$ \\
Volumen de operación (m. de cultivo) & 0,011 & $\mathrm{~m}^{3}$ \\
Volumen de operación (biomasa) & 0,010 & $\mathrm{~m}^{3}$ \\
Altura total & 0,54 & $\mathrm{~m}$ \\
Altura de operación (m. de cultivo) & 0,43 & $\mathrm{~m}$ \\
Altura de operación (biomasa) & 0,39 & $\mathrm{~m}$ \\
Diámetro & 0,18 & $\mathrm{~m}$ \\
Volumen máximo de biomasa & 0,012 & $\mathrm{~m} 3$ \\
\hline
\end{tabular}


LÍNEA DE INVESTIGACIÓN: BIOTECFUA

- Potencia de las bombas. Los resultados de los cálculos para las dos propuestas de diseño se encuentran en la Tabla 6. Como se observa la potencia requerida de las bombas para las dos propuestas de diseño tienen valores que oscilan entre 1,16 E-9 y 1,49 E-8 caballos de fuerza, una potencia muy pequeña comparada con las que se encuentra en el mercado, esto se debe principalmente a la baja velocidad del flujo. Adicionalmente se observó que las pérdidas de energía están influenciadas principalmente por la diferencia de los valores de las alturas entre los puntos de referencia; esto se ve evidenciado cuando se comparan las potencias de las bombas de carga de la primera propuesta la cual tiene mayor diferencia de altura entre los dos puntos de referencia y la segunda. Sin embargo, al tener que pasar los flujos con una alta precisión, debido al crecimiento del microorganismo, la adecuación de una bomba es necesario.

Cuando se comparan las potencias de las bombas calculadas con el trabajo de Torres y Zambrano cuyos resultados son de 1,64E-5 hp y 1,65E-5 hp para las bombas de carga y descarga respectivamente, se observa que, aunque son mayores que las calculadas en este proyecto, siguen siendo bajas y aun así ellos utilizaron bombas para su proceso, por lo tanto, se necesita el uso de las bombas para suplir las necesidades de las microalgas.

Tabla 6.

Potencia de las bombas de carga y descarga para las dos propuestas

\begin{tabular}{cccccc}
\hline Propuesta & Bomba & $\begin{array}{c}\text { Pérdida total } \\
\text { de energía }(\mathbf{m})\end{array}$ & $\begin{array}{c}\text { Carga total } \\
\text { agregada }(\mathbf{m})\end{array}$ & $\begin{array}{c}\text { Potencia agregada } \\
\text { por la bomba (kW) }\end{array}$ & $\begin{array}{c}\text { Potencia } \\
\text { agregada por la } \\
\text { bomba (hp) }\end{array}$ \\
\hline 1 & $4,31 \mathrm{E}-5$ & $1,41 \mathrm{E}-1$ & $1,11 \mathrm{E}-8$ & $1,49 \mathrm{E}-8$ \\
\hline $\begin{array}{l}\text { Bomba de } \\
\text { carga } \\
\text { Bomba de } \\
\text { descarga } \\
\text { Bomba de } \\
\text { carga } \\
\text { Bomba de } \\
\text { descarga }\end{array}$ & $2,53 \mathrm{E}-5$ & $6,10 \mathrm{E}-2$ & $4,80 \mathrm{E}-9$ & $6,44 \mathrm{E}-9$ \\
\hline & $4,63 \mathrm{E}-5$ & $9,12 \mathrm{E}-2$ & $7,18 \mathrm{E}-9$ & $9,62 \mathrm{E}-9$ \\
\hline
\end{tabular}

\section{Ventajas y desventajas de las propuestas de diseño.}

- Análisis cualitativo. Se observó que algunas de las propuestas tienen desventajas y ventajas con respecto a la otra.

Primera propuesta: Tiene como ventajas el uso de accesorios móviles como la llave bilateral para la salida del flujo del fotobiorreactor y la llave de muestra, la entrada por la parte superior al tanque de biomasa. No obstante, esta propuesta, tiene desventajas como la salida lateral de la manguera del tanque de almacenamiento, porque puede provocar pérdida del medio de cultivo que queda por debajo de esta salida.

Segunda propuesta: Tiene la ventaja de contar con accesorios móviles. En el diseño se tuvieron en cuenta los parámetros como esterilidad y estabilidad estructural del sistema como o más importantes. En este sentido, se pensó en una estructura para soportar los tanques de almacenamiento; 
la salida posterior del tanque de alimentación, para evitar el desperdicio de medio de cultivo; y los microfiltros, con el fin de evitar la contaminación del medio del cultivo y de la biomasa.

- Análisis Cuantitativo: La potencia de las bombas, para el caso de las dos propuestas, cambia con las diferencias de altura, definidas por el volumen de operación de los tanques de almacenamiento del medio de cultivo; estos volúmenes de operación están directamente relacionados con el tiempo de retención en los tanques, debido a que a mayores tiempos de retención, mayores son las diferencias de altura entre los puntos de referencia, y así mismo, mayor será la potencia que requerirá la bomba para funcionar. Esto se evidencia con el análisis de la potencia de las bombas de carga y descarga en las diferentes propuestas. Por ejemplo, la potencia de la bomba de carga de la primera propuesta es de 9,62E-9 hp con una diferencia de altura de 0,091 $\mathrm{m}$, es menor a la segunda propuesta cuya potencia de la bomba es de 1,49E-8 hp con una diferencia de altura de $0,141 \mathrm{~m}$. Hay que aclarar que los flujos, tanto de entrada como de salida del fotobiorreactor y el diámetro en las dos propuestas, se mantuvieron constantes. No obstante, si se disminuye el diámetro de la tubería, mayores serán las pérdidas de energía a través de esta. Por lo tanto, teniendo en cuenta las ventajas presentadas, la propuesta que se recomienda es la segunda.

Nota: En este trabajo la operación inicial es por lotes y conforme el cultivo se acerca a la fase estacionaria se empieza a operar en sistema continuo. No obstante, teniendo en cuenta que los valores de estrés generados por el sistema son bajos y la microalga Chlorella vulgaris es resistente a fuerzas de cizalla, se puede manejar un sistema semi-continuo con mayores flujos, disminuyendo los costos de algunos equipos y facilitando su adquisición.

\section{CONCLUSIONES}

De las variables analizadas en el presente estudio, se observó que las variables operacionales: flujo y tiempo de retención, tienen mayor importancia para el diseño de este sistema, que los parámetros del cultivo.

Los flujos de entrada y salida están influenciados por la velocidad de crecimiento de la microalga, y este a su vez dependerá de las condiciones y el tiempo que se hayan considerado para el cultivo; en consecuencia, las velocidades de crecimiento tomarán valores diferentes.

Por otro lado, se encontró que los valores de las potencias de las bombas en cada propuesta de diseño dependen de la diferencia de alturas entre los puntos de referencia y debido a que el caudal manejado es bajo, es irrelevante para este tipo de sistemas, lo que permitió modificar las propuestas sin tener un cambio significativo de este valor.

\section{REFERENCIAS}

Abalde, J. (1995). Parámetros de cultivo. En J. Abalde, Microalgas: Cultivo y aplicaciones (págs. 19-63). España: Universidad de Coruña. 
LÍNEA DE INVESTIGACIÓN: BIOTECFUA

Ángel Cruz, M. C., \& Pimienta Blanco, W. Y. (2013). Incidencia del nitrógeno en la producción de biomasas y ácidos grasos de la microalga Chlorella vulgaris en un fotobiorreactor de panel plano a escala laboratorio. Revista de Investigación Universidad de América, 7-18.

Bruno, D. F., Mota, A., Teixeira, J. A., \& Vicente, A. A. (2015). Continuous cultivation of photosynthetic microorganisms: Approaches, applications and future trends. Biotechnology Advances, $1228-1245$.

Caicedo, L. A., \& Rodríguez Laiton, S. E. (2016). Obtención de biomasa y azúcares reductores a partir de microalgas producidas en un fotobiorreactor airlift a escala laboratorio. Bogotá: Fundación Universidad de América.

Cho, Dae-Hyun, et al. Influence of limiting factors on biomass and lipid productivities of axenic Chlorella vulgaris in photobioreactor under chemostat cultivation. En: Bioresource technology.vol. 211, p. 367

Doran, P. M. (1995). Principios de ingeniería de los bioprocesos. España: Acribia.

Ganuza, E., \& Izquierdo, M. (2007). Lipid accumulation in Schizochytrium G13/2S produced in continuous culture. Appl Microbiol Biotechnol, 985-990.

López, T., Malagón, M., \& Torres, C. (2013). Evaluación de desempeño del fotobiorreactor Airlift para la producción de biomasa y lípidos a escala banco. Bogotá, Colombia: Universidad de América.

Michels, M., van der Goot, A., Nosrsker, N., \& Wijffels, R. (2010). Effects of shear stress on the microalgae Chaetoceros muelleri. Bioprocess and biosystems engineering, 921-927.

Montouto, A. (2010). Diseño de una planta piloto para bioconversion del glicerol procedente de la industria de los biocombustibles. Cádiz, España: Universidad de Cádiz.

Mott, R. L., Enriquez, J., \& León, J. (2006). Mecánica de fluidos. México: Pearson Educación.

Osorio, R., \& Zapata, M. (04 de noviembre de 2004). Practica 3. Densidades de Líquidos. Obtenido de Universidad de Antioquia: http://docencia.udea.edu.co/cen/tecnicaslabquimico/02practicas/ practica03.htm

Romdhane, F. E. (2012). Development and validation of a minimal growth medium for recycling Chlorella vulgaris culture. Bioresource Technology, 366.

Shih-Hsin, H., Xiaoting, Y., Tomohisa, H., Jo-Shu, C., \& Akihiko, K. (2014). Perspectives on engineering strategies for improving biofuel production from microalgae $-\mathrm{A}$ critical review. Biotechnology Advances, 1448-1459.

Steffe, J., \& Daubert, C. (2006). Ductos y tuberías para Bioprocesos: Reologia y análisis. Still valley Drive: Freeman press. 